Journal of Engineering and Applied Sciences 7 (3): 295-299, 2012

ISSN: 1816-949X

(C) Medwell Journals, 2012

\title{
A New Methodology for Handling Empty Zone Problem over Mobile Ad Hoc Network
}

\author{
S. Kannan, V.P. Arunachalam and S. Karthik \\ SNS College of Technology, Coimbatore, Tamil Nadu, India
}

\begin{abstract}
Group communications are important in Mobile Ad hoc Networks (MANET). Multicast is an efficient method for implementing group communications. However, it is challenging to implement multicast packet forwarding over a dynamic topology. Researchers propose a novel Efficient Geographic Multicast Protocol (EGMP). A network wide zone based bi-directional tree is constructed to achieve more efficient multicast delivery. The position information is used to guide the zone structure building, multicast tree construction and multicast packet forwarding which efficiently reduces the overhead for route searching and tree structure maintenance. Several strategies have been proposed to further improve the efficiency of the protocol, for example introducing the concept of zone depth for building an optimal tree structure and integrating the location search of group members with the hierarchical group membership management. Finally, researchers design a scheme to handle empty zone problem faced by most routing protocols using a zone structure. The scalability and the efficiency of EGMP are evaluated through simulations. The simulation results demonstrate that EGMP has high packet delivery ratio and low control overhead and multicast group joining delay under all test scenarios and is scalable to both group size and network size. EGMP has significantly lower control overhead, data transmission overhead and multicast group joining delay.
\end{abstract}

Key words: MANET, EGMP, multicasting, zone, dynamic topology, India

\section{INTRODUCTION}

An ad hoc network is composed of mobile nodes without the intervention of any fixed infrastructure or central administration. It is also called Mobile Ad hoc Networks (MANETs), they are characterized by dynamic topology due to node mobility. Disaster relief, conferences, action directions given to the soldiers in a battlefield, communications required during a rescue operation, the support of multimedia games and teleconferences are some examples of these applications. With a one-to-many transmission pattern, multicast is an efficient method to realize group communications. Using multicast instead of sending through multiple unicasts not only minimizes link consumption but also reduces sender and router processing, communication costs and delivery delay.

Conventional MANET multicast protocols can be ascribed into two main categories, tree based and mesh based. However due to the constant movement as well as frequent network joining and leaving from individual nodes, it is very difficult to maintain the tree structure using these conventional tree-based protocols (e.g., MZRP, MZR). The mesh-based protocols (e.g., FGMP, ODMRP) are proposed to enhance the robustness with the use of redundant paths between the source and the destination pairs (Fig. 1).

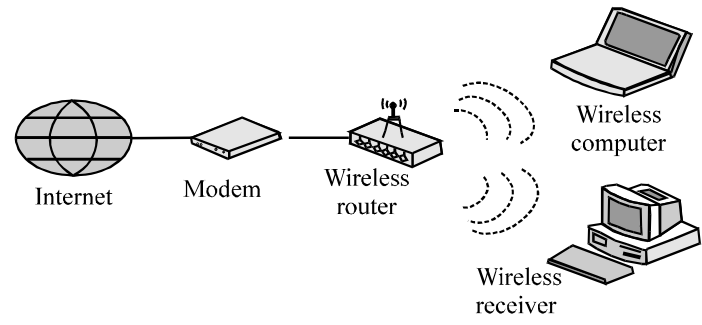

Fig. 1: MANET approaches

Conventional multicast protocols generally do not have good scalability due to the overhead incurred for route searching, creation and maintenance of the tree/mesh structure over the dynamic MANET. A straight-forward way to extend the geography-based transmission from unicast to multicast is to put the addresses and positions of all the members into the packet header, however the header overhead will increase significantly as the group size increases which constrains the application of geographic multicasting only to a small group (Fig. 2).

Besides requiring efficient packet forwarding, a scalable geographic multicast protocol also needs to efficiently manage the membership of a possibly large group, obtain the positions of the members and build routing paths to reach the members distributed in a possibly large network terrain. In this research,

Corresponding Author: S. Kannan, SNS College of Technology, Coimbatore, Tamil Nadu, India 


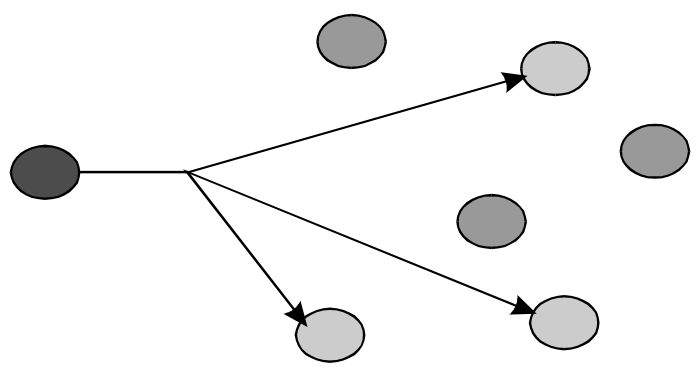

Fig. 2: Multicasting

researchers propose an efficient geographic multicast protocol, EGMP which can scale to a large group size and large network size. The protocol is designed to be comprehensive and self-contained, yet simple and efficient for more reliable operation. Instead of addressing only a specific part of the problem, it includes a zone based scheme to efficiently handle the group and efficiently track the locations of all the group members without resorting to an external location server. By making use of the location information, EGMP could quickly and efficiently build packet distribution paths and reliably maintain the forwarding paths in the presence of network dynamics due to unstable wireless channels or frequent node movements (Karthik et al., 2010).

MANET characteristics: The fundamental difference between fixed networks and MANET is that the computers in a MANET are mobile. Due to the mobility of these nodes, there are some characteristics that are only applicable to MANET. Some of the key characteristics are described (Karthik et al., 2010):

Dynamic network topologies: Nodes are free to move arbitrarily, meaning that the network topology which is typically multi-hop, may change randomly and rapidly at unpredictable times.

Bandwidth constrained links: Wireless links have significantly lower capacity than their hard wired counterparts. They are also less reliable due to the nature of signal propagation.

Energy constrained operation: Devices in a mobile network may rely on batteries or other exhaustible means as their power source. For these nodes, the conservation and efficient use of energy may be the most important system design criteria.

The MANET characteristics imply different assumptions for routing algorithms as the routing protocol must be able to adapt to rapid changes in the network topology.
Applications of MANET: There are numerous scenarios that do not have an available network infrastructure and could benefit from the creation of an ad hoc network (Karthik et al., 2010).

Rescue/Emergency operations: Rapid installation of a communication infrastructure during a natural/ environmental disaster that demolished the previous communication infrastructure.

Law enforcement activities: Rapid installation of a communication infrastructure during special operations.

Commercial projects: Simple installation of a communication infrastructure for commercial gatherings such as conferences, exhibitions, workshops and meetings.

Educational classrooms: Simple installation of a communication infrastructure to create an interactive classroom on demand.

Military battlefield: Ad hoc networking would allow the military to take advantage of common place network technology to maintain an information network between the soldiers, vehicles and military information head quarters.

Commercial sector: Emergency rescue operations (like fire, flood, earthquake, etc.) must take place where non-existent or damaged communications infrastructure and rapid deployment of a communication network is needed.

Local level: Ad hoc networks can autonomously link an instant and temporary multimedia network using notebook computers or palmtop computers to spread and share information among participants at a conference or classroom.

\section{MATERIALS AND METHODS}

In this study, researchers summarize the basic procedures assumed in conventional multicast protocols and then introduce a few geographic multicast algorithms proposed in the literature. EGMP uses a location-aware approach for more reliable membership management and packet transmissions and supports scalability for both group size and network size. As the focus of the study is to solve the empty zone problem by merging the zones. Researchers introduce packet retransmission by selecting the next path from the routing table (Xiang et al., 2011). In DSM, each node floods its location in the network. A 
source constructs a steiner tree and encodes the multicast tree into each packet and delivers the packet by using source routing. LGT requires each group member to know the locations of all other group members and proposes two overlay multicast tree: a bandwidth-minimizing LGS tree and a delay-minimizing LKG tree (Xiang et al., 2006).

In PBM a multicast source node finds a set of neighboring, next hop nodes and assigns each packet destination to one next hop node. The next hop nodes, in turn, repeat the process. Thus, no global distribution structure necessary. EGMP in attempts to build a more efficient multicast tree through a centralized calculation for tree construction as is more applicable for smaller group. The focus of EEGMP; however it is to solve the empty zone problem and to improve the efficiency of geometric multicast ( $\mathrm{Ji}$ and Corson, 2001).

The HRPM and SPBM are more related to the research, as they also support hierarchical group management. HRPM consists of two key design ideas hierarchical decomposition of a large group into hierarchy of recursively organized manageable-sized subgroups and the use of distributed geographic hashing to construct and maintain such a hierarchy.

Problem statement: Empty zone problem faced by most routing protocols using a zone structure. Zone may become empty when all the nodes move away from it. Packet moving from source to reach the destination, it fails to reach the destination then it takes longer waiting time to send that packet retransmit.

Efficient geographic multicast protocol: Some of the notations to be used are:

Zone: The network terrain is divided into square zones as shown in Fig. 3.

r: Zone size, the length of a side of the zone square. To reduce intra-zone management overhead, the intra-zone nodes can communicate directly with each other without the need of any intermediate relays.

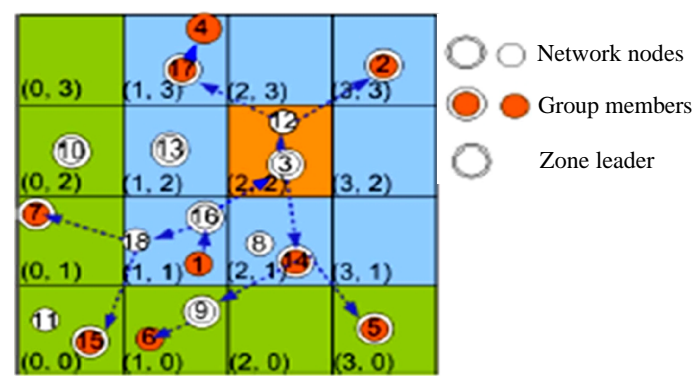

Fig. 3: Zone structure and multicast session example
Zone ID: The identification of a zone. A node can calculate its zone ID (a, b) from its position coordinates $(\mathrm{x}, \mathrm{y})$ as: $\mathrm{a}=\left[\mathrm{x}_{\mathrm{i}} \mathrm{xOr}\right], \mathrm{b}=(\mathrm{y} ; \mathrm{yOr})$ where $(\mathrm{x} 0 ; \mathrm{y} 0)$ is the position of the virtual origin which can be a known reference location or determined at network setup time.

zLdr (Zone leader): A zLdr is elected in each zone for managing the local zone group membership and taking part in the upper tier multicast routing (Xiang et al., 2011, 2007).

Tree zone: The zones on the multicast tree. The tree zones are responsible for the multicast packet forwarding. A tree zone may have group members or just help forward the multicast packets for zones with members.

Root zone: The zone where the root of the multicast tree is located.

Zone depth: The depth of a zone is used to reflect its distance to the root zone. Enhance Efficient Geographic Multicast Protocol (EGMP) to solve empty zone problem by merging the zones. Last node in a particular zone is zone leader before moving to the next zone it merges with its neighboring zone there by reducing the overhead in the zone.

Introducing packet retransmission by selecting the next path from the routing table. If a packet send from source to destination gets fail, the existing system again find the shortest path to retransmit the packet and there is longer waiting time.

To avoid the longer waiting time, researchers find the next shortest path with help of routing table to transmit the packet (Ji and Corson, 2001; Gui and Mohapatra, 2007; Devarapalli and Sidhu, 2001).

Zone structure: The zone-structure is virtual and calculated based on a reference point. Therefore, the construction of zone structure does not depend on the shape of the network region and it is very simple to locate and maintain a zone. The zone is used in EGMP to provide location reference (Woo and Singh, 2001).

Zone leader: A zone leader is elected through the cooperation of nodes and maintained consistently in a zone. When a node appears in the network, it sends out a beacon announcing its existence. When a node appears in the network, it sends out a beacon announcing its existence. Then it waits for an Intvalmax period for the beacons from other nodes (Gui and Mohapatra, 2004; Transier et al., 2007). 
Every intvalmin a node will check its neighbor table and determine its zone leader under different cases. The neighbor table contains no other nodes in the same zone, it will announce itself as the leader. The flags of all the nodes in the same zone are unset which means that no node in the zone has announced the leadership role. If the node is closer to the zone center than other nodes, it will announce its leadership role through a beacon message with the leader flag set.

About $>1$ node in the same zone have their leader flags set, the one with the highest node ID is elected. Only one of the nodes in the zone has its flag set then the node with the flag set is the leader.

Moving between different zones: When a member node moves to a new zone, it must rejoin the multicast tree through the new leader. When a leader is moving away from its current zone, it must handover its multicast table to the new leader in the zone, so that all the downstream zones and nodes will remain connected to the multicast tree.

\section{RESULTS AND DISCUSSION}

Researchers implemented the EGMP protocol using NS2 (Network Simulator) library and compare it with ODMRP which is widely used and considered to be robust over a dynamic network.

Effect of the network size: To study the scalability of the protocol with network size, researchers varied the network range from $1500 \times 1500$ to $3900 \times 3900 \mathrm{~m}$. The node density is kept as before thus the total number of nodes is varied from 156-1056 nodes. Since, the periodic local and network-wide message flooding in SPBM saturates the memory faster, researchers run simulations on SPBM with the network size increasing up to only $3300 \times 3300 \mathrm{~m}$ with 756 nodes.

EGMP has a better scalability to the network size than ODMRP and SPBM as demonstrated. The delivery ratios of ODMRP and SPBM drop faster than that of EGMP with the increase of network size. When the network size reaches $3900 \times 3900 \mathrm{~m}$ with 1056 nodes, the difference between the delivery ratios of ODMRP and EGMP is $>55 \%$ (Fig. 4).

Effect of the group size: Next researchers evaluate the protocol performance with the group size varied from 10-200 members. Demonstrates that EGMP can scale to a large group size and perform well with various group sizes. When the group size increases, the delivery ratios of EGMP rise (Fig. 5).

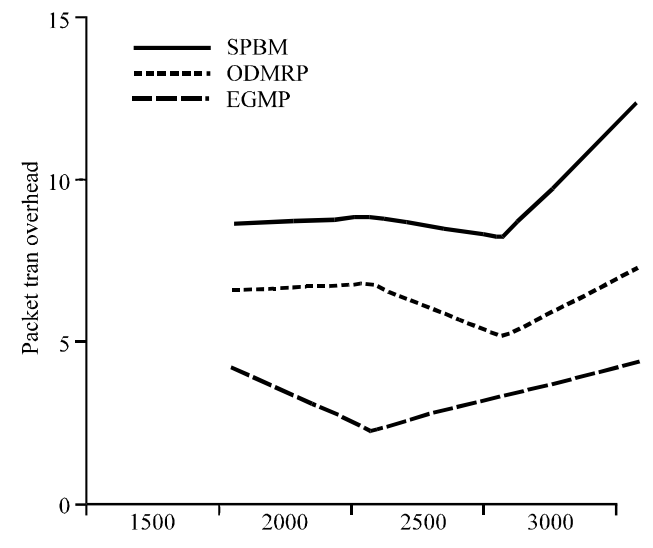

Fig. 4: Performance vs. network size

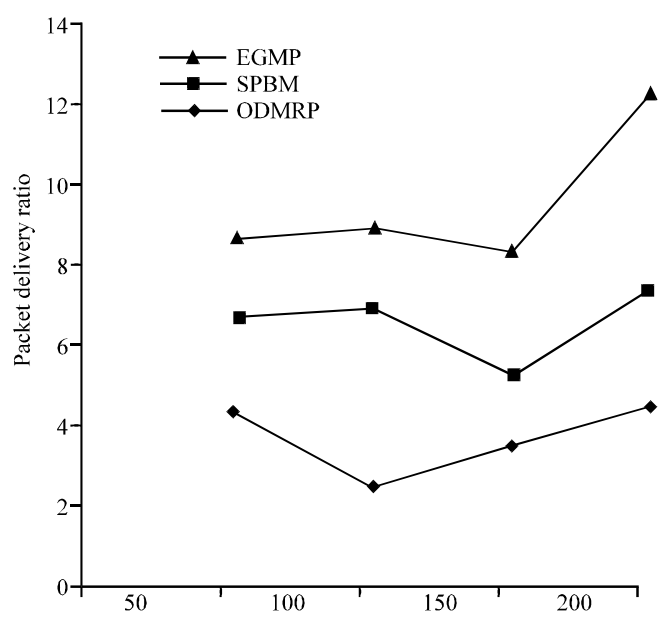

Fig. 5: Performance vs. group size

\section{CONCLUSION}

The empty zone problem will be eliminated by merging the zones and the packet retransmission will be made more efficiently by selecting the route with the help of simulation tool. EGMP significantly reduces the tree construction and maintenance overhead and enables quicker tree structure adaptation to the network topology change. Researchers also develop a scheme to handle the empty zone problem which is challenging for the zone based protocols. Additionally, EGMP makes use of geographic forwarding for reliable packet transmissions and efficiently tracks the positions of multicast group members without resorting to an external location server. The simulation results demonstrate that EGMP has high packet delivery ratio and low control overhead and multicast group joining delay under all cases studied and is scalable to both the group size and the network size. Compared to the geographic multicast protocol SPBM, it has significantly lower control overhead, data transmission overhead. 


\section{REFERENCES}

Devarapalli, V. and D. Sidhu, 2001. MZR: A multicast protocol for mobile ad hoc networks. Proceedings of the IEEE International Conference on Communications, June 11-14, 2001, Helsinki, Finland, pp: 886-891.

Gui, C. and P. Mohapatra, 2004. Scalable multicasting for mobile Ad Hoc networks. Proceedings of the IEEE INFOCOM 23rd AnnualJoint Conference of the IEEE Computer and Communications Societies, March 711, 2004, Hong Kong, pp: 2119-21 29.

Gui, C. and P. Mohapatra, 2007. Overlay multicast for MANETs using dynamic virtual mesh. Wirel. Networks, 13: 77-91.

$\mathrm{Ji}, \mathrm{L}$. and M.S. Corson, 2001. Differential destination multicast-A MANET multicast routing protocol for small groups. Proceedings of the IEEE INFOCOM, April 22-26, 2001, IEEE, pp: 1192-1202.

Karthik, S., S. Kannan, V.P. Arunachalam and T. Ravichandran, 2010. An investigation about performance comparison of Multi-Hop Wireless Ad-Hoc network routing protocols in MANET. Int. J. Comput. Sci., Vol. 7.
Transier, M., H. Fubler, J. Widmer, M. Mauve and W. Effelsberg, 2007. A hierarchical approach to position-based multicast for mobile ad-hoc networks. Wirel. Networks, 13: 447-460.

Woo, S.C.M. and S. Singh, 2001. Scalable Routing Protocol for Ad Hoc Networks. Wirel. Network, 7: $512-529$.

Xiang, X., X. Wang and Z. Zhou, 2006. An efficient geographic multicast protocol for mobile Ad Hoc networks. Proceedings of the 2006 International Symposium on on World of Wireless, Mobile and Multimedia Networks, June 26-29, 2006, BuffaloNiagara Falls, pp: 8-82.

Xiang, X., Z. Zhou and X. Wang, 2007. Robust and scalable geographic multicast protocol for mobile Ad Hoc networks. Proceedings of the IEEE INFOCOM 26th IEEE International Conference on Computer Communications, May 6-12, 2007, Anchorage, pp: 2301-2305.

Xiang, X., X. Wang and Y. Yang, 2011. Supporting efficient and scalable multicasting over mobile Ad Hoc networks. IEEE Trans. Mobile Comput., Vol. 10. 10.1109/TMC.2010.176. 